\title{
CareStore Platform for Seamless Deployment of Ambient Assisted Living Applications and Devices
}

\author{
S. Wagner, F.O. Hansen, C.F. Pedersen, \\ M. Memon, F.H.Aysha \\ Aarhus University \\ Aarhus, Denmark
}

\author{
M. Mathissen, C. Nielsen, \\ O.L. Wesby \\ Sekoia ApS \\ Aarhus, Denmark
}

\begin{abstract}
A large number of ambient assisted living technologies coexist without a common language between them. They have different user interfaces, communication infrastructures and maintenance plans, which limit the inclusion of efficiencyenhancing solutions in the home care sector. Also, deployment of applications and devices are overly complex, and cannot be handled by the healthcare staff or citizens themselves, but most often require support from technical personnel. This increases cost and reduces the feasibility of implementing new technologies. Following a participatory design approach the CareStore project investigated the feasibility of creating an open and flexible infrastructure for facilitating seamless deployment and integration of assisted living devices and applications on heterogeneous platforms without requiring intervention from technical staff. A range of limitations and challenges are identified, and a solution is suggested.
\end{abstract}

Ambient assisted living, pervasive healthcare, participatory desgin, infrastructure, Continua Alliance, healthcare standards.

\section{INTRODUCTION}

Europe is currently witnessing a demographic shift with a rising population of seniors that is causing significant pressure on the national healthcare systems in Europe and beyond [1,2]. In 2015, there will be more than 75 million people above 65 years of age living in Europe. This is expected to increase the healthcare expenses significantly, compelling European governments to invest in new technologies and solutions that may assist European seniors in living more independent lives while reducing healthcare expenditures at the same time. Current state-of-the-art technology in this area is limited to proprietary, or de-facto proprietary, solutions that will only support hardware and software provided by a single or a limited number of vendors [3]. This includes products from Bosch [4], Tunstall [5], Intel [6], and several other vendors [3]. A private or public care facility, such as a nursing home, runs the risk of vendor-lock-in when choosing such a platform. To exemplify, when choosing the Tunstall Telehealth monitor as the primary platform for the residents and nursing staff, the nursing home can only deploy technology and software supported by Tunstall. If products from other vendors are needed, another system infrastructure needs to be deployed, possibly resulting in several parallel or "silo" systems [3]. Installation and support is most often provided by separate support organisations, making deployment of telemedicine and ambient assisted living equipment difficult and expensive [7]. Intel's Continua Alliance relies on IEEE 11073 standards as a platform for co-developing software interfaces to support devices from all Continua Alliance compliant vendors [8]. However, this is limited to biomedical devices, and does not allow for deploying third party software applications. Also, integration with electronic healthcare records is not always feasible to achieve, as only the platform vendor is typically allowed to achieve such integration. Opposing this, general purpose platforms, such as Linux, Windows, and Android, are fully open for both software and hardware deployment. However, these solutions may be perceived as too open, posing intolerable security risks to the residents and staff at the care facilities. Furthermore, today no easy deployment mode is available for state-of-the-art care facility devices on such general purpose platforms.

Support technicians must typically perform the installation, which is not economically viable in a large scale context. To exemplify this; a wireless automatic blood pressure device or a medical weight scale cannot easily be installed in the home of a citizen, and data must often be manually entered into the electronic healthcare record system. Furthermore, the ability to quickly attach a new device to a specific resident in a plug-andplay fashion is not solved by any vendors at present.

As the cost of acquiring advanced healthcare devices and infrastructure has become negligible, it may be argued that the main challenge is now an organizational and political challenge rather than economical or technical. The willingness of enduser organizations to acquire new technology appears hampered by unclear channels for encompassing new technology.

Several research projects have suggested conceptual and technical platforms and frameworks for facilitating ambient assisted living (AAL) devices and applications. However, most of these have not matured to become operational beyond the experimental and prototypic stages. These include UniversAAL [9], OpenAAL [10], SOPRANO [11], Hydra [7], OpenCare [3], and several others [12]. So far, these systems have not gained widespread support by the European healthcare community and institutions, beyond research funded projects.

The aim of the CareStore project is to investigate the potential of introducing a common platform for seamless deployment of healthcare and AAL devices and applications in the home of citizens in need of care. This paper reports on the findings from the initial field studies and preliminary conceptual and technical design considerations. Here, the main objectives were to investigate why novel AAL technology is only slowly penetrating the gap from research to real world 
usage, identify the barriers and how these may be overcome, and evaluate a conceptual and technical model for overcoming these challenges through a participatory design approach and cooperation with the pervasive healthcare community.

\section{RELATED WORK}

The majority of AAL related research studies are trying to achieve interoperability and integration [13]. Others studies focus on medical device communication and electronic health record standards $[8,14]$. Besides, wireless sensor networks are also investigated for AAL usage [15]. Others realize the importance of accuracy in personal health monitoring [16]. Quality attributes including usability, security and safety are also considered as key requirements for AAL environments [17-19]. Conceptual frameworks and design methodologies provide experience and guidance for better modeling and implementation of AAL systems [3,7-12].

A number of general purpose and platform specific application stores exist: Apple AppStore, Google Play, and Windows Store. While these platforms could possibly be adapted to allow for specific care applications and drivers, they do not presently support the deployment of physical drivers. Linux features the package deployment system, which could feasibly also be adapted, but lacks a financial transactional model. The UniversAAL uStore [9] also seeks to address related issues, featuring a marketplace for promoting and finding applications. The uStore project is also based on open source and backed by a consortium of several universities and private enterprises. However, the uStore project relies solely on the UniversAAL framework and is not open for other frameworks.

\section{MATERIALS AND METHODS}

\section{A. Methods}

We initially surveyed the literature and state of the art commercial and research AAL platforms and standards in order to identify limitations and challenges to be addressed. Then, following an explorative research agenda we planned a three phase study consisting of a range of field studies at several Danish care facilities including three nursing homes and one rehabilitation care facility, as well as a German care facility group, in the first phase. Also, we visited two general practice clinics, with five general practitioners each, as well as three hospital departments and outpatient clinics. In the second phase, we arranged several workshops in cooperation with enduser organizations and relevant stakeholders including seniors, medical doctors, nurses, physiotherapists, and chronic patients, as well as individual interview sessions with stakeholders, both staff and residents, as well as patients in their home setting. The planned third phase involves the future deployment of the initial CareStore prototype at several care facilities. First phase was carried out as a purely observational descriptive study, with the aim of understanding the problem domain. The second phase included presenting possible solutions to the identified challenges during the first phase, and elaborating on these in a participatory design inspired manner. The suggested solution, presented in the next section, is being developed iteratively as a proposal to increase proliferation of novel AAL technologies by simplifying the installation process for staff and residents, striving for "seamless" operation. It is currently based on the results of the participatory design process and is still considered work-in-progress as we expect it to further evolve during the CareStore project's life cycle.

\section{B. Suggested solution}

The overall objective of the CareStore project is to develop an inexpensive and user-friendly open-source platform that will allow all vendors free and easy access to publish new devices and applications, as well as supporting staff and residents' easy deployment of such devices and applications in residential apartments, without requiring technical staff intervention.

Figure 1: Overview of the CareStore system

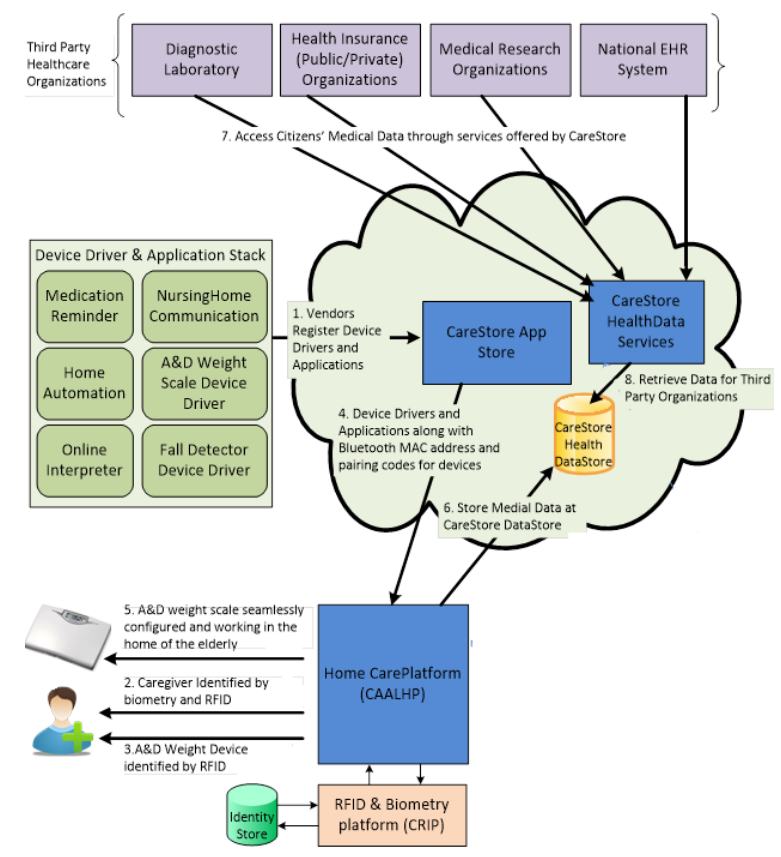

The CareStore project focuses on creating a research platform for evaluation of the impact of introducing such seamless deployment of AAL applications and devices in the care facilities. The solution consists of the following three main components (see Figure 1 for an overview):

1. CAALHP: The Common Ambient Assisted Living Home-Platform is an open-source runtime execution platform for reliable and secure execution of services, applications, and healthcare device drivers, as well as a user-interface for the staff and residents.

2. CRIP: The Common Recognition and Identification Platform is used for identifying staff, residents, and devices using RFID and biometrics-based recognition and identification technologies

3. CareStore Marketplace: Is an online shared platform for uploading and storing healthcare and AAL drivers and applications, as well as for allowing CAALHP and CRIP to download drivers and applications in a seamless fashion. 


\section{RESUlts}

\section{A. Identified limitations and challenges}

From the initial survey of the field we identified the following limitations pertaining to existing commercial state of the art AAL platforms:

- Existing platforms are proprietary and partially closed. Typically only a limited list of third party sensors can be integrated, and typically it is not possible to integrate with third party care applications

- With existing platforms it is only possible to use a limited set of third party devices, often excluding e.g. non IEEE 11073 PHD compliant devices, unless adapted manually

- Server data communication is restricted to limited platforms, making it difficult to integrate to national, regional, or municipal-operated backend systems and electronic patient records

- Existing platforms do not offer seamless integration of new devices and applications but require technical assistance during installation and deployment

\section{B. Limited use of existing state of the art technology}

We found that the proliferation of existing state of the art AAL technology was very low at the visited care facilities. Only a few devices were available and most were not connected to any relevant system or infrastructure. While all of the care facilities had an electronic care record system, not a single care facility had any devices that could procure relevant data for automatic data collection, such as a blood pressure device or a medical weight scale. One of the visited care facilities utilized a sensor device for detecting a senile patient getting out of bed and sounding an alert. However, this sensor was not connected to an overall infrastructure or platform, but rather relied on a stand-alone infrastructure.

\section{Openness for change}

We found that staff and residents at nursing homes and private homes would welcome novel AAL solutions and systems, if they were sufficiently user friendly to install and operate. In general, there was much annoyance with current technologies not being able to interoperate and being too complex. For instance, blood pressure and weight measurements were typically measured in the room of the patient, and the data later manually typed into the healthcare record of the patient. This was found to be very unsatisfactory and potentially error prone by staff.

\section{Difficulty with installation of new devices and training}

During the first phase we found that staff and residents at the care facilities and private homes typically perceived the installation of new devices to be overly complex and unfeasible for work-use. This was by most considered as an unsurpassable barrier to introducing novel technology, unless the devices were installed and maintained by technical staff. As no such technical support organization was in place at the visited municipal-based and private care facilities, there was low willingness to experiment with novel solutions. Although most care staff members could possibly be trained to be self-reliant, the high throughput of nurses and other caregivers at all of the visited care facilities, meant it would be very difficult to keep all staff members updated.

\section{E. Concerns for equipment costs and vendor lock-in}

We also found that leading staff members had concerns with the traditional high expenditures of novel technologies which could only rarely be justified by resulting perceived benefits and optimizations. Also, there were concerns whether novel equipment would be compatible with existing client devices and computers, or whether the care facility would have to invest in yet another system and infrastructure, implying additional staff training and maintenance plans. Staff in general requested that devices and systems should be able to easily interface, and that it should be possible to install devices and applications from different vendors in an independent and seamless manner.

\section{DISCUSSION}

\section{A. Adressing identified limitations and challenges}

The CareStore project aims at implementing the suggested solution, and evaluate its ability to address the limitations and challenges identified and reported in this paper. If successful, the outcome of the project could be used to support the daily work tasks of healthcare professionals at care facilities and in the private senior homes, but more importantly, it may be used by the research community as a general purpose evaluation tool for investigating whether a better infrastructure, allowing for more seamless deployment of devices and applications, will actually lead to a higher adaption and acceptance of novel ambient assisted technologies. This evaluation process is planned in the third and final phase of the CareStore project. Here, the suggested solution will be introduced into the clinical setting, first at a German care facility with 250 residents, and then at several Danish and European care facilities, in order to evaluate the solutions impact, usability, applicability, and feasibility by deploying the technology to comparable residential care apartments at the same care facility in a randomized and blinded study. Also, another group of staff and residents using the same healthcare and AAL devices that are not relying on the CareStore platform's support for seamless deployment technology, and a third group without technology support, will be used as controls. This should allow us to evaluate the impact of the suggested CareStore platform solution, when combined with relevant care technologies.

However, in order to meet these objectives, it is important that the pervasive healthcare community will support this initiative by participating actively. The CareStore project in itself is not relevant to evaluate without being used in conjunction with the appropriate healthcare and AAL devices. As a consequence, it is necessary to attract and motivate a sufficient number of AAL and healthcare device vendors to support and embrace CareStore standards. Without sufficient momentum and "critical mass" of relevant supported applications and devices, the CareStore project is not likely to be able to provide the needed documentation of effect and will itself become a "silo" project. Thus, the CareStore consortium aims at integrating with as many healthcare vendors as possible, including offering to make adjustments of programming interfaces and standards to suit external needs. 
Only by joining hands and cooperating will we be able to realize the full CareStore vision. This includes cooperating with related initiatives, such as the UniversAAL and other panEuropean projects and research groups. This process has already been initiated, through a comprehensive review of AAL systems, platforms, and technologies, as well as healthcare standards, for a detailed mapping of the field.

\section{B. Planned CareStore Contributions:}

The CareStore project aims at identifying the needed improvements with regard to usability, availability, integration, interoperability, scalability, security, and management of AAL and pervasive healthcare technologies, and evaluating the impact through in situ usage. The following components are planned for this evaluation study:

- A mechanism for automatic and seamless deployment of devices and applications with the objective of preventing elderly citizens and staff from having to handle the technically challenging tasks of device detection, installation, and configuration, in order to improve usability

- An implementation of a marketplace for providing access to download device drivers and applications with the objective of increasing availability

- Integration with the national Danish healthcare infrastructure, Net4Care, and similar emerging European integration infrastructures, allowing data created at the home platform to be shared by third party organizations

- A component for automatic discovery and integration with different types of healthcare and AAL devices including IEEE 11073

- Security features for ensuring that data is always encrypted and signed, by authenticating staff, patients, and devices using biometry and/or RFID

- Securing flexibility and openness by allowing external stakeholders to deploy independent applications and manage their own systems including users and devices.

\section{Conclusion}

The technical difficulties of deploying novel AAL technologies and devices to care facilities and private homes appear to be the main barrier for the proliferation of this technology to a broader audience. The CareStore project will enable us to investigate the effects of more seamless deployment. However, this potential has yet to be determined, and will require the pervasive healthcare and related communities to cooperate in order to obtain the needed "critical mass" of supported healthcare devices, applications, and services.

\section{ACKNOWLEDGMENT}

The CareStore Project is funded by the European Commission as part of the Seventh Framework Program. Grant agreement no. 315158. Thank you to the many students and colleagues working on the initial conceptual models and feasibility prototypes of the CareStore project. Thank you to the staff and residents at the visited care facilities. Also, thank you to the other members of the CareStore consortium.

\section{REFERENCES}

[1] European Commision. "Europe's population is getting older. How will this affect us and what should we do about it?". 200517 March;IP/05/322.

[2] J. Keye, T. Zitzelberge. "Overview of healthcare, disease, and disability". In: Bardram J, Mihailidis A, Wan D, editors. "Pervasive Computing in Healthcare”. CRC Press, 2006. p. 3-20.

[3] S. Wagner and C. Nielsen. "OpenCare project: An open, flexible and easily extendible infrastructure for pervasive healthcare assisted living solutions." Proceedings of the 3rd International Conference on Pervasive Computing Technologies for Healthcare Pervasive Health 2009, April 1, 2009 - April 3; London, United kingdom: IEEE Computer Society.

[4] Bosch Healthcare. "Health Buddy". Available at: http://www.boschtelehealth.com/en/us/products/health_buddy/health_buddy.html. Accessed 1.1.2011.

[5] Intel Corporation. "Intel Health Guide PHS6000". Available at: http://www.intel.com/corporate/healthcare/emea/eng/healthguide/pdfs/H ealth_Guide_Product_Brief.pdf. Accessed 1.1.2011.

[6] Tunstall Limited. "Telehealth solutions". Available at: http://www.tunstall.co.uk/Our-products/Telehealth-solutions. Accessed 01.01.2011.

[7] M. Eisenhauer, P. Rosengren, P. Antolin. "HYDRA: a development platform for integrating wireless devices and sensors into ambient intelligence systems". Internet of Things New York, NY: Springer Verlag; 2010. p. 367-373.

[8] R. Carroll, R. Cnossen, M. Schnell, D. Simons. "Continua: An interoperable personal healthcare ecosystem". IEEE Pervasive Computing 2007:90-94.

[9] M.R. Tazari, F. Furfari, Á.F. Valero, S. Hanke, O. Höftberger, D. Kehagias, et al.” The universAAL Reference Model for AAL”. 2012.

[10] P. Wolf, A. Schmidt, J. P. Otte, et al. "OpenAAL - The Open Source Middleware for Ambient-Assisted Living (AAL)". 2010.

[11] P. Wolf, A. Schmidt and M. Klein. "SOPRANO - An extensible, open AAL platform for elderly people based on semantical contracts”. 3rd Workshop on Artificial Intelligence Techniques for Ambient Intelligence (AITAmI'08), 18th European Conference on Artificial Intelligence (ECAI 08), Patras, Greece: Citeseer; 2008.

[12] Demiris G. Smart homes and ambient assisted living in an aging society. New opportunities and challenges for biomedical informatics. Methods Inf Med 2008;47(1):56-57.

[13] G. Worsley, "Assisted Living Innovation Platform- Dallas and interoperability”, www.raate.org.uk/ uploaded/Graham\%20Worsley.pdf, 2011.

[14] J. D. Trigo, et. al., :Standard-compliant real-time transmission of ECGs: Harmonization of ISO/IEEE 11073-PHD and SCP-ECG”, IEEE Conference, Engineering in Medicine and Biology Society, 2009.

[15] D. Zubiete et. al., "Review of wireless sensors networks in health applications”, IEEE Conference, Engineering in Medicine and Biology Society, 2011.

[16] A. J. Jara, M. A. Zamora, et. al., “An internet of things---based personal device for diabetes therapy management in ambient assisted living (AAL)". Personal and Ubiquitous Computing, 15 (4) 431-440, 2011.

[17] P. O. Antonino, D. Schneider, et. al., "Evaluation of AAL Platforms According to Architecture-Based Quality Attributes", Ambient Intelligence - 2nd Int. Joint Conference on AmI, 2011.

[18] C. Doukas, I. Maglogiannis, et. al., "Enabling data protection through PKI encryption in IoT m-Health devices," 12th Int. Conf. on Bioinformatics \& Bioengineering (BIBE), 25,29, 2012.

[19] S. F. Whitehead and J.M. Goldmann, "Connectivity to Improve Patient Safety”, http://www.psqh.com/januaryfebruary-2010/390-connectivityto-improve-patient-safety.html, 2010. 